\title{
EL MATERIALISMO DE MARX. REFLEXIONES METODOLÓGICAS ACERCA DE LA ONTOLOGIA MARXISTA EN EL CAPITAL
}

Voy a desarrollar uno de los temas que estimo más intrigantes en la historia de las relaciones entre la obra de Marx y la escuela de pensamiento marxista. Me refiero a la ontología que manejó este científico y revolucionario, especialmente en su escrito magno, El Capital, en el que esta doble condición se materializa al efectuar el análisis crítico de la estructura económica del sistema capitalista, de tal modo que la corrección del análisis implica, simultáneamente, la crítica del sistema.

Marx, como fundador del materialismo histórico, ha sido tradicionalmente presentado como materialista puro, sin que esta cualidad haya sido puesta bajo sospecha.

Pero, ¿es esto así? ¿Responde esta clasificación ontológica a la verdad histórica sobre el pensamiento de Marx? ¿O se trata, una vez más, de una lectura sintomática, pero en sentido inverso a la explicada por Althusser, que efectúan los discípulos de un científico con la obra de su maestro? Lectura sintomática inversa, pues no se trata de leer, como lo indica Althusser, y sin duda es factible hacerlo, en los intersticios de la obra escrita para desentrañar su auténtico sentido, leer lo no escrito, pero que grita en el texto, sino ; de borrar, como si no existieran, afirmaciones que fueron fundamentales para el primer expositor del paradigma en cuestión! $\mathrm{Al}$ igual que en una ceguera selectiva se leen e, inmediatamente, se suprimen-reprimen, de la lectura, proposiciones completas. Represión que juzgo similar a la freudiana, pues se "olvida" lo acontecido, sin dar, ni creer que deba darse, explicación racional alguna de la supresión. Es más, se suprime y se niega, al mismo tiempo, la supresión.

Fin física, los Principia de Newton explicitan, con todas sus 
letras, que la mecánica clásica está diseñada de forma tal $-\mathrm{y}$ esto comprende, entre otros elementos, el insospechado principio de inercia- que el movimiento sólo pudo iniciarse, y mantenerse, si se apela a una deidad suprema como primer impulsor del movimiento, y su constante alimentador. El teísmo de Newton - para éste, consustancial e inseparable de su teoría- fue suprimido-reprimido por sus seguidores, a tal punto que exponerlo viene a ser, en ocasiones, una excentriciỏad de historiador de la ciencia. La mecánica clásica fue, efectivamente, desarrollada por los seguidores de Newton sin este supuesto teísta. La evolución que experimentó hasta Laplace, y su reformulación en términos de cálculo diferencial, lo hicieron "innecesario" -Dios es una hipótesis innecesaria, escribió Laplace a Napoleón-y, por consiguiente, olvidable. La mecánica clásica devino el paradigma de la ciencia "positiva".

Voy a señalar una situación equivalente en la obra de Marx, que, hasta donde conozco, ha sido escasamente mencionada en la literatura marxista, y cuya resolución, tal como se produjo con el teísmo de la física newtoniana, no ha sido encarada en forma consecuente.

Mi estrategia va a consistir en mostrar el problema, explicar las razones que tuvo Marx para encarar una solución tan problemática, exponer a continuación un intento reciente de librar a la teoría marxista de ésta, y marcar las insuficiencias de que adolece, para proponer, finalmente, una reformulación de la teoría del valor que haga innecesaria la solución ontológica de Marx.

\section{El problema ontológico en el primer capítulo de FI Capital}

Paso a citar in extenso a Marx:

Tomemos otras dos mercancías, por ejemplo el trigo y el hierro. Sea cual fuere su relación de cambio, éste se podrá representar siempre por una ecuación en la que determinada cantidad de trigo se equipara a una cantidad cualquiera 
de hierro, por ejemplo: 1 quarter de trigo $=a$ quintales de hierro. (¿Qué denota esta ecuación? Que existe algo común, de la misma magnitud en dos cosas distintas, tanto en 1 quarter de trigo como en a quintales de hierro. Ambas, por consiguiente, son iguales a una tercera, que en sí y para sí no es ni la una ni la otra. Cada una de ellas, en tanto valor de cambio, tiene que ser reducible a esa tercera. ( $E l$ Capital, México: Siglo XXI, 1979, Tomo I, vol. I, pp. 4647. El subrayado me pertenece.)

La lectura correcta no permite equívocos: aquí, Marx dice que sólo se pueden comparar magnitudes de cosas distintas, si poseen una cosa, que no es ni hierro ni trigo pero que, desde el punto de vista ontológico, tiene su mismo status. Más adelante dirá: "Ese algo común que se manifiesta en la relación de intercambio o en el valor de cambio de las mercancías, es, pues, su valor.' (Op. cit., p. 47.)

Fl valor es, entonces, una cosa, una sustancia. El primer apartado del capítulo primero de El Capital, habla, precisamente, de la sustancia del valor (ibid., p. 43).

Si es una sustancia, ¿de qué clase de sustancia se trata?, ¿cómo aparece en la mercancía, oculta a la vista y, sin embargo, tan visible para la teoría?

Marx aclara:

Ahora bien, si ponemos a un lado el valor de uso del cuerpo de las mercancías, únicamente les restará una propiedad: la de ser productos del trabajo. No obstante, también el producto del trabajo se nos ha transformado entre las manos. Si hacemos abstracción de su valor de uso, abstraemos también los componentes y formas corpóreas que hacen de él un valor de uso. Ese producto ya no es una mesa o casa o hilo o cualquier otra cosa útil. Todas sus propiedades sensibles se han esfumado. Ya tampoco es producto del trabajo del ebanista o del albañil o del liilandero o de cualquier otro trabajo productivo determinado. Con el carácter útil de los productos del trabajo se desvanece el carácter útil de los trabajos representados en ellos $\mathbf{y}$, por 
ende, se desvanecen también las diversas formas concretas de estos trabajos; éstos dejan de distinguirse, reduciéndose en su totalidad a trabajo humano indiferenciado, a trabajo abstractamente humano (ibid., pp. 46-47, subrayado mío).

Analicemos cuidadosamente: la maniobra consiste en quitar de la mercancía su materialidad, aquello que la compone, su forma corpórea. Un idealista contestaría que si quito a algo todas sus propiedades sensibles, queda algo, su esencia. Esta respuesta parece impensable para un materialista, o simplemente un anti-esencialista. Marx sostiene que esta maniobra de abstracción no es una simple abstracción del análisis que no influye al objeto en cuestión, sino una abstracción real y material, similar a la que se produce en un alambique cuando se destila carbón, o alguna otra sustancia: impensa damente, este análisis de abstracción real deja un sustrato material que estaba escondido tras el duro exterior de la cosa, un alcaloide volátil adherido a las paredes de la probeta. Pero no hay maniobra empírica para contrastar la presencia de esta sustancia exótica, el valor. Sólo una maniobra teórica de abstracción.

Hoy, y también en la época de Marx, un materialista se rebelaría contra esta supuesta sustancia fundante, que es independiente y distinta de la materia real de las cosas. Supongo los sudores fríos que habrá sentido Marx al escribir estas líneas; no en balde la redacción del primer capítulo de El Capital la emprendió, como confiesa, infinitas veces, y ésta no debe de haber sido una de las dificultades menores çue enfrentó.

El ejemplo del alambique no es enteramente gratuito; poco más adelante, nos dirá que luego de su maniobra de abstracción real ha quedado un residuo, y que este residuo es el valor:

Fxaminemos ahora el residuo de los productos del trabajo. Nada ha quedado de cllos salvo una misma objetividad espectral, una mera gelatina de trabajo humano indiferenciado, esto es, de gasto de fuerza de trabajo humana sin consideración a la forma en que se gastó la misma. Fsas cosas tan sólo nos hacen presente que en su producción se empleó fuerza humana de trabajo, se acumuló 
trabajo humano. Fn cuanto cristalizaciones de esa sustancia social común a ellas, son valores (ibid., p. 47. El subrayado es mío).

Leamos rectamente a Marx. Aquí no se trata de ninguna figura literaria, de ninguna paráfrasis por interpretar. Las mercancías en su intercambio tienen algo de común, este algo es una sustancia, aquella que queda luego de eliminar la materialidad de la mercancía concreta. El valor es una sustancia, trabajo humano que se cristaliza. La naturaleza de esta sorprendente sustancia es tan extraña como la de aquella que se desprende del cuerpo cuando éste muere, y algo permanece; Marx no vacila en llamarla, al igual que en el caso de la muerte corporal, objetividad espectral, pues sólo el espectro de algo permanece luego que su corporeidad se anula.

El trabajo, por consiguiente, no sólo crea nuevas formas de la materia, creando un universo humanizado, a la medida del hombre -y esto es lo que se aceptaría sin discusión en cualquier me dio marxista-, sino que además se cristaliza, sc materializa él mismo en la mercancía; crea, en una palabra, nueva materia. Y esto es más arduo de aceptar hoy y en la época de Marx.

Un valor de uso o un bien, por ende, sólo tiene valor porque en él está objetivado o materiälizado trabajo abstractamente humano (ibid., p. 47. Nuevamente, el subrayado es mío).

\section{Los motivos de Marx}

Estos párrafos, y otros desperdigados en el primer capítulo de El Capital, son escasamente señalados en la literatura marxista como motivo de análisis o de preocupación teórica. Se los lee sin lecrlos, para pasar a lo supuestamente relevante, el estudio de la plusvalía, y el resto de las categorías económicas. Sin embargo, Marx, que reescribió varias veces este primer capítulo, los mantuvo hasta su publicación; pensaba que no cran eliminables, que sin ellos su fundamentación de la teoría 
del valor perdía coherencia; o, más bien, que no había tal fundamentación.

Llegamos aquí a un punto crucial. Marx creía que sólo podía poner en una ecuación de igualdad dos cosas disímiles, dos mercancías distintas, tal como hierro y trigo, si y sólo si ellas poseían algo en común. Su teoría entera del valor dependía de poder establecer esta ecuación. Obviamente, aquello que tienen en común es la cantidad de trabajo humano abstracto, como lo aclara páginas más adelante. Por consiguiente, el trabajo humano abstracto debía ser una cosa medible, y aquello que poseían las mercancías en común. Observemos que estamos ante un típico problema metodológico, que Marx resuelve en forma ontológica. La disyuntiva que ahora se abre no debió ser de decisión fácil. Marx debía optar entre suponer que el trabajo en la mercancía era una sustancia distinta a la materia, ontologizar el trabajo, con lo que su obra tendría dos sustancias, la materia y el trabajo, o mantener a ultranza su monismo ontológico materialista. Opta, coherente con toda su trayectoria intelectual, por esta última solución: El Capital debía ser expresión de un monismo materialista. Pero la solución dista de ser satisfactoria: una sustancia-materia fantasmal, materia que surge de la nada -el trabajo creando materia-, materia que queda cuando eliminamos la materia. Esta solución coja, de una materia que casi no es materia, debió parecerle preferible a un dualismo ontológico.

Que se trata realmente de poder fundamentar la igualdad entre mercancías intercambiables, que poseían igual valor, y no en principio de la noción de valor, lo podemos constatar leyendo la versión que ofrece del "problema de Aristóteles" con respecto al valor, y que Marx hace suya.

Dice, en efecto:

5 lechos = una casa

no difiere de

5 lechos $=$ tanto o cuanto dinero. 
Aristóteles advierte además que la relación de valor en la que se encierra esta expresión de valor, implica a su vez el hecho de que la casa se equipare cualitativamente al lecho, y que sin tal igualdad de esencias, no se podría establecer una relación recíproca, como magnitudes conmensurables, entre cosas que para nuestros sentidos son diferentes. "EI intercambio, dice, no podría darse sin igualdad, la igualdad a su vez, sin la conmensurabilidad." Pero aquí se detiene perplejo, y desiste de seguir analizando la forma del valor. "En verdad es imposible que cosas tan heterogéneas sean conmensurables", esto es, cualitativamente iguales. Esta igualación no puede ser algo extraño a la verdadera naturaleza de las cosas, y por consiguiente un mero "arbitrio para satisfacer la necesidad práctica".

El propio Aristóteles nos dice, pues, por falta de qué se malogra su análisis ulterior: por carecer del concepto de valor. ¿Qué es lo igual, es decir, cuál es la sustancia común que la casa representa para el lecho, en la expresión del valor de éste? Algo así "en verdad no puede existir", afirma Aristóteles. ¿Por qué? Contrapuesta al lecho, la casa representa un algo igual, en la medida en que esto representa en ambos - casa y lecho- algo que es efectivamente igual. Y eso es el trabajo humano.

El genio de Aristóteles brilla precisamente por descubrir en la expresión del valor de las mercancías una relación de igualdad. Sólo la limitación histórica de la sociedad en que vivía le impidió averiguar en qué consistía, en verdad, esa relación de igualdad (ibid., pp. 73-74).

Vemos aquí resumidos los puntos que queríamos destacar en la argumentación marxista sobre el valor:

i) la necesidad de fundamentar la relación primordial de igualdad entre mercancías,

ii) la fundamenta una sustancia común, una esencia común, iii) esta esencia, esta sustancia es el trabajo humano, una materia sui generis. 
Intuyo que es en las ciencias naturales donde Marx toma apoyo en su fundamentación. Voy a citar dos párrafos para señalar luego una coincidencia que no creo casual.

El primero ya ha sido citado; es cuando expone que es ". . una mera gelatina de trabajo indiferenciado". El segundo, dice, reafirmando: "Por primera vez este mismo valor se manifiesta auténticamente como una gelatina de trabajo indi. ferenciado"' (p. 77). La figura de la sustancia del valor como gelatina se reitera, en un autor de una inmensa riqueza $e x$. presiva. Pienso que la reiteración obedece a motivaciones más profundas que una mera carencia de imaginación.

Por la misma época, los físicos, armados de la teoría newtoniana, y además filósofos materialistas, se encuentran ante un problema que paso a resumir: los astros se atraían gravitatoriamente, pese a la distancia que los separaba. Un materialista sólo podía aceptar la acción de una fuerza que se ejerciera entre partículas espacialmente próximas: la materia transmitía la fuerza. En el caso del sistema planetario, y del universo, la fuerza de atracción se ejercía a distancia, y esto era impensable desde el punto de vista materialista de los siglos XVIII y XIX. Inventan entonces, para eliminar la acción a distancia, una sustancia que llena los intersticios del espacio, y a cuyo través se transmite la fuerza; nace el éter, que se va a mantener como "sustancia" casi hasta principios de nuestro siglo, para luego desaparecer de la ciencia. Pues bien, el éter era imaginado como una gelatina de sustancia. La pregunta sobre si el éter se comportaba como gelatina o como fluido fue resuelta a favor de su naturaleza gelatinosa.

Qué mejor para Marx que atribuir a su materia, que no es materia, la misma gelatinosidad que poseía el etéreo éter.

Tenemos expuesto entonces el problema de Marx, que es un problema metodológico, de fundamentación teórica del valor, y cuya solución no creía tan inaccptable, ya que la ciencia por antonomasia, la física, había recurrido a nociones similares. Desaparecido el éter hace casi un siglo, y bien entendido que la teoría del valor no está en juego en esto, sino sólo su fundamentación, rechazo la solución de Marx, con el mismo espíritu con que Marx la habría rechazado; para 
exponer una fundamentación que elimine su ontología dudosa, a favor de un claro monismo ontológico.

Marx creía, con los científicos de su época, que todo término teórico tenía una contrapartida ontológica, es decir, que cada término de una teoría describía algo. Esto, y suponer que sólo podía comparar cosas disímiles si poseían algo en común, lo lleva a una ontología materialista espúrea, en la que coexisten materia por un lado, y materia espectral por el otro.

Entremos de lleno a estas dificultades de fundamentación para superarlas con herramientas forjadas en el último siglo.

\section{La solución Werner Diederich y Hans F. Fulda}

Fstos autores intentan un análisis estructural de $E l$ Capital, y sobre todo, del primer capítulo, usando el aparato teórico desarrollado por Joseph Sneed en The Logical Structure of Mathematical Physics. Voy a dejar a un lado las consideraciones sobre la completa reconstrucción estructural, para referirme exclusivamente a las reflexiones de estos autores sobre la ley del valor, ya que su reconstrucción elimina el dispositivo ontológico montado por Marx.

Al establecer la ley, piensan que está dicho todo lo que hay que decir sobre valor, pues de acuerdo con esta metateoría, los términos teóricos encuentran su definición en el uso funcional que les asigna la teoría.

La ley del valor es presentada de la siguiente manera:

$$
\begin{aligned}
& \text { (LV) } \quad \mathrm{z} \# \mathrm{v} \# \mathrm{p} \quad \text { (Léase: el trabajo determina el valor, } \\
& \text { el valor determina el precio.) }
\end{aligned}
$$

Aquí, z es el tiempo de trabajo socialmente necesario para producir cualquier mercancía; $p$ es su precio.

Tanto $z$ como $p$ son funciones sobre los números reales positivos; se trata de funciones no teóricas en la terminología snecdiana, ya que para su determinación no hace falta tomar en cuenta la teoría marxista; en efecto, el precio y el tiempo de trabajo son nociones cuya magnitud puede medirse sin que 
en esa medida medie la teoría de Marx. En cambio, $v$ es una función que sólo puede determinarse si se acepta la teoría. \# es una función entre $z, v$ y $p$, tal que esa función, conociendo $z$ determina el valor, y conociendo el valor determina el precio.

Hasta aquí, muy resumi da a efecto de la discusión presente, la solución Diederich-Fulda.

Como pretenden acertadamente estos autores, elimina to da ontología innecesaria, y a que se trata de funciones matemáticas sobre los números reales. Si la reconstrucción fuera correcta, el problema de Marx se habría disuelto.

Voy a señalar un inconveniente muy grave de esta ley del valor propuesta que me lleva a desecharla.

Una función determina $v$ partiendo de $z$, y otra función determina $p$ partiendo de $v$. Pero en esta formulación, $v$ es eliminable. En efecto, es posible establecer una función \#\# tal que aplicada sobre $z$ determine $p$, sin pasar por la magnitud intermedia del valor.

Se conectaría, me diante una función, un término no-teórico con otro término no-teórico, sin ninguna necesidad del constructo marxista:

$$
\text { z \#\# } \mathbf{p} \text {. }
$$

La reconstrucción propuesta, en una consecuencia lógica inmediata, lleva a eliminar el núcleo teórico de la ley del valor. Si esto es así, sólo cabe rechazar la teoría marxista por superflua, o rechazar la reconstrucción que permite tal cosa.

Pienso que la reconstrucción no hace justicia al pensamiento de Marx, y por consiguiente opto, entre la metateoría y la teoría, por la teoría marxista. Mi labor, en adelante, va a consistir en proponer una definición adecuada del valor que sea compatible tanto con El Capital, como con una posible reconstrucción estructural alternativa.

\section{El valor sin metafísica}

Volvamos al procedimiento de leer sin prejuicios el texto de Marx, a fin de extraer de él los conceptos tal cual figuran, la 
problemática tal cual es, no aquella que no leemos, ni aquella que nos figuramos debiera estar y no está.

Si hacemos esto, nuevamente nos embarga la extrañeza. No en balde han fracasado los intentos de definir valor. No es ésta la tarea que se propuso Marx. Éste en ningún momento intenta definir valor, sino -y sostengo que esto es una novedad metodológica que sólo cincuenta años después va a tener un paralelo en ciencias formales- lo que va a intentar definir es igual valor.

Leamos a Marx:

El valor consiste en la relación de intercambio que media entre tal cosa y cual otra, entre tal medida de un producto social y cual medida de otro (citado por Marx en la p. 45).

Sea cual fuere su relación de cambio, ésta se podrá representar siempre por una ecuación en la que determinada cantidad de trigo se equipara a una cantidad cualquiera de hierro, por ejemplo: 1 quarter de trigo $=a$ quintales de hierro.

En el intercambio, por consiguiente, siempre podremos establecer una ecuación que exprese igualdad entre cierta cantidad de una mercancía, con otra cantidad de otra mercancía. Esta ecuación establece que cada uno de los miembros tiene igual valor.

Aquí surge el problema que Marx intenta resolver por vía ontológica, ya que supone que:

Para averiguar de qué manera la expresión simple del valor de una mercancía se encierra en la relación de valor entre dos mercancías, es necesario, en un principio, considerar esa relación con total prescindencia de su aspecto cuantitativo. Por regla general se procede precisamente a la inversa, viéndose en la relación de valor tan sólo la proporción en que se equiparan determinadas cantidades de dos clases distintas de mercancías. Se pasa por alto, de esta suerte, que las magnitudes de cosas diferentes no llegan a ser comparables cuantitativamente sino después de su reducción a la misma unidad. Sólo en cuanto expresiones de 
la misma unidad son magnitudes de la misma denominación, y por lo tanto, conmensurables.

Fl razonamiento metodológico pudiera ser considerado impecable: la ecuación de igualdad entre el hierro y el trigo es sólo posible si puede establecerse una medida común entre ambos, su valor, que es igual. Y establecer el valor igual, es anterior a la situación cuantitativa de establecer la magnitud del valor, y de las mercancías que entran en la ecuación.

Falla Marx al suponer que sólo puede fundamentar la igualdad sobre bases ontológicas. Su gran acierto es mantener, a riesgo de materialidades fantasmales, la primacía de la igualdad, y de la noción de valor que la establece.

Hice mención, al pasar, de las ciencias formales. En la historia de las matemáticas, una pregunta similar a la de Marx va a recibir una respuesta cuya fundamentación se aparta de lo ontológico.

Gottlob Frege, en Los fundamentos de la aritmética (México: U.N.A.M., 1972), se interroga sobre qué es el número, o qué significa el símbolo 1 ; descarta que el número pertenezca a las propiedades de las cosas externas, o que sea algo subjetivo. Fiel a uno de los principios básicos que guían sus investigaciones, aquel que dice: "no se debe preguntar por el significado de una palabra aislada, sino en el contex to de una proposición", parte del curso de pensamiento que expone consiste en mostrar las proposiciones en cuyo seno el símbolo numérico encuentra significado. Cita, como corroboración de su punto de vista, a Spinoza: "respondo que una cosa sólo con respecto a su existencia, pero no a su esencia, puede ser llamada una o única; ya que sólo nos representamos las cosas por medio de números, una vez que las hemos reducido a un módulo común. Por ejemplo, quien tiene en la mano un tálero y un sestercio no pensará en el número dos si no puede cubrir este tálero y este sestercio con uno y el mismo nombre, a saber, monedas: después puede afirmar que tiene dos monedas, pucsto que con el nombre moneda no sólo designa al sestercio, sino también al tálero". Spinoza habla de cubrir dos objetos con el mismo nombre, y en ningún momento de en- 
contrarles una sustancia común. Esto se debe, lo explica Frege, a que los objetos no son depositarios del número, el número no está en las cosas, sino que son los conceptos los portadores de los números, y lo que el número indica es cuántos objetos caen bajo un concepto.

En sus palabras: "La apariencia, surgida de algunos ejemplos anteriores, de que a la misma cosa corresponderían números distintos, se aclara por cuanto que ahí se tomó a los objetos como portadores del número. Tan pronto como instituimos en su derecho al verdadero portador, el concepto, los números se muestran tan excluyentes como en su campo los colores."

¿Qué clase de proposiciones va a encontrar Frege para definir un predicado ubicuo como el número, fundante por ello de las matemáticas, tal como el valor, tan ubicuo como el número, lo es de la economía?

Piensa que:

En nuestro caso debemos definir el sentido de la proposición "el número que corresponde al concepto $\mathrm{F}$ es el mismo que corresponde al concepto G" (p. 170).

Más adelante expresa:

No obstante, aún queda una duda. Una proposición de reconocimi ento siempre debe tener un sentido. Si ahora entendemos la posibilidad de coordinar biunívocamente los objetos que caen bajo el concepto $F$ con los que caen bajo el concepto G como una igualdad, al decir: "el número que corresponde al concepto $\mathrm{F}$ es igual al número que corresponde al concepto G", y con ello introducimos la expresión "el número que corresponde al concepto $F$ ", entonces obtenemos un sentido para la igualdad sólo si ambos lados tienen la forma justamente mencionada. De acuerdo con tal definición, no podríamos juzgar si una igualdad es verdadera o falsa en caso de tener únicamente un lado de esta forma. Esto nos dio pie para la definición:

El número que corresponde al concepto $\mathrm{F}$ es la extensión 
del concepto "concepto equinumérico respecto al concepto F", con lo cual llamamos a un concepto F equinumérico respecto a un concepto $\mathrm{G}$ si existe la mencionada posibilidad de coordinación biunívoca.

Aquí, se presupone que se conoce el sentido de la expresión "extensión del concepto". Este modo de superar la dificultad ciertamente no encontrará consenso general, y muchos preferirían allanar tal dificultad de otra manera. Yo tampoco pongo un peso decisivo en lo atrayente de la extensión de un concepto (p. 205).

No fue nuestro propósito seguir los vericuetos de la argumentación de Frege para intentar responder a la pregunta "¿qué es un número?" Los párrafos citados pertenecen a la anteúltima página de su escrito, $\mathrm{y}$ son un buen resumen de sus conclusiones.

V eámoslos: primero comienza por el sentido de una proposición de igualdad, la cual, para establecerse, debe ser producto de la correspondencia biunívoca entre ambos términos de la ecuación. Ahora puede decir qué cosa sea hablar de número en uno de los miembros de la igualdad: es la extensión que posee el concepto "concepto equinumérico respecto al con. cepto F".

Frege termina haciendo algunas reflexiones sobre lo arduo y poco aceptable de su solución.

Quiero hacer notar que, en principio, el problema de Frege es similar al problema de Marx, ya que en ambos se trataba de establecer el sentido de una ecuación de igualdad, una referida a números, otra a valores.

La solución de Frege va a ser adoptada por B. Russell, quien ofrece una formulación alternativa.

Este autor pone en un conjunto los apóstoles de Jesucrito, en otro los mariscales de Napoleón; para establecer un signo igual entre ambos conjuntos, hace corresponder miembro a miembro cada uno de los elementos de cada conjunto; efectúa, al igual que Frege, una correspondencia biunívoca entre los miembros de los conjuntos. Ahora, dice, tienen el mismo número. Hasta ese momento va a contestar qué es un núme- 
ro: es el conjunto de todos los conjuntos que tienen ese mismo número. Con la introducción del concepto "tener el mismo número", y construir un conjunto de todos los conjuntos entre los cuales se puede efectuar la correspondencia biunívoca, Russell elude la "extensión del concepto" fregeana, para obtener una definición de la noción de número.

Antes de proponer una solución Frege-Russell a la definición del valor, quisiera comentar previamente la posibilidad de trasladar esta discusión matematica al seno de las ciencias fácticas. Independientemente de aceptar que, en principio, las matemáticas constan de proposiciones cuya elucidación no puede ser indiferente para la elucidación de otras proposiciones, en otros términos, que la claridad conceptual que aportan pueda ser trasladada a otros ámbitos, voy a comentar un problema de fundamentación física, cuya resolución siguió parámetros como los anteriormente descritos para el número. Me refiero a la definición de masa por E. Mach. Seguiré la exposición que de éste hace Ulises Moulines en "La génesis del positivismo en su contexto científico", Diánoia, México: F.C.E., 1975.

Comienza Mach por rechazar, por oscura, la identificación común de la masa con la "cantidad de materia": "La idea de la cantidad de materia no es adecuada para determinar y aclarar el concepto de masa, puesto que ella misma carece de suficiente claridad."

En vez de eso, parte Mach del tercer principio de Newton (acción $=$ reacción) para determinar primeramente la igualda d de masas; define, entonces, primeramente el concepto de igualdad de masa, y luego el concepto general de masa como magnitud: "Decimos que dos cuerpos tienen igual masa si, cuando interactúan, se inducen mutuamente aceleraciones iguales y de sentido opuesto; con ello no hemos hecho más que denominar una relación fáctica."

De allí pasa a la definición de masa en general, introduciendo una escala de masas. Por convención, escoge una de las clases de igualdad de masas y asigna a sus elementos cl valor unidad. Para determinar la masa de cualquier otro cuerpo $\mathrm{C}$ no perteneciente a dicha clase, se toma un representante 
A de ella, y se coloca en proximidad espacial de C. Observamos sus aceleraciones mutuas y definimos: "C tiene la masa $m$ si y sólo si la aceleración inducida en $A$ por $C$ es $m$ veces mayor que la aceleración inducida en C por A". Concluye Mach: "mi definición resulta del intento de determinar las relaciones de dependencia entre los fenómenos, y de eliminar toda oscuridad metafísica".

Esta obra de Mach es anterior a los escritos de Frege-Russell. Con todo, no deja de ser interesante cómo, libre ya de la necesidad de la sustancia común que deben poseer ambos miembros de una igualdad, puede hablar de igual masa simplemente si ciertos efectos son iguales. Salta por sobre la definición de masa, pues efectivamente no la formula: de igual masa, pasa a instrucciones para cuantificar masas.

Veamos a continuación si podemos aplicar estos antece. dentes en fundamentación de las matemáticas y la física a nuestro problema marxista.

Habíamos partido del mismo punto que Marx: la ecuación de la forma simple del valor, que se expresaba por una igualdad entre cantidades $x$ y $y$ de distintas mercancías, del tipo:

$$
x \text { mercancía } \mathrm{A}=y \text { mercancía } \mathrm{B}
$$

ejemplificada como 20 varas de lienzo $=1$ chaqueta.

Estamos casi a la misma altura, con el simple escrito de Marx, en lo que es fruto de una labor de fundamentación en Mach, quien debe partir de los principios de Newton para construir la noción de igualdad de masas. Aquí está dicho desde el arranque: las distintas mercancías tienen el mismo valor. Aunque no lo diga con estas palabras textuales, Marx define la noción "tener igual valor". Esto es una relación empírica, tal como la propone Mach con la masa; empíricamente, dos cantidades $x, y$ de dos mercancías tienen el mismo valor cuando efectivamente se intercambian en esa proporción.

Marx parte del hecho empírico del intercambio de mercancías en el seno de un modo de producción. Constata que en el curso del intercambio se establecen igualdades empíri- 
cas del tipo de 1 chaqueta $=20$ varas de tela, y al igual que Frege, comprende que el valor que puedan tener como objetos de intercambio, no pertenece a las características intrínsecas de la tela o la chaqueta. La igualación empírica podría fundamentarse en el simple hecho aparencial de ser objetos intercambiables. No es éste el camino que elige Marx. Existe una acción previa al intercambio, la producción del objeto en cuestión por el trabajo humano; éste es el hecho primario. Es el trabajo el que va a permitir el intercambio - sin trabajo no existe mercancía-, y el que, por consiguiente, le va a dar su valor; el intercambio no es de ninguna manera azaroso, pues su condición de posibilidad es que ambas mercancías tengan igual valor, y éste se cuantificará midiendo la duración del trabajo.

Pero el valor no es sólo definible en el ámbito del trabajo; si intentáramos decir esto, sería un error. En el acto de la producción, no podemos todavía hablar de valor; debemos hacerlo sólo cuando la producción se intercambia como mercancía (en una sociedad de autoconsumo, se producen bienes de uso, mas no valor), y entonces, lo correcto sería decir que tienen igual valor.

Veamos cómo la maniobra es similar a la de Frege-Russell, aunque su complejidad sea mayor, ya que aquí el campo empírico, la economía, presenta una complejidad mayor que el restingido campo de las matemáticas: la complejidad que va de lo formal a lo empírico. Una acción real, el contar, engendradora del número, permite igualar colecciones de objetos reales que posean igual número. Otra acción real, el trabajo, formadora de valor, permite intercambiar colecciones de objetos -con lo que se establece su igualdad-que tengan igual valor.

De aquí en adelante divergen las soluciones de Marx y de Russell. El primero piensa que sólo se pueden igualar dos conjuntos si poseen una sustancia en común; el trabajo, por consiguiente, genera una sustancia que existe en la mercancía, además de su materialidad, y que sólo se pone de manifiesto en el intercambio. El valor es una sustancia. Ignoro las raíces de esta convicción metodológica de Marx; no debe ser ajena a 
ello el hecho de que en su época todos los científicos aceptaban sin dudar un instante la sustancia real, aunque oculta a los sentidos, detrás de todos los términos teóricos. En nuestros días pensamos que ello no es necesario; algunos términos teóricos referirán a funciones matemáticas, como es el caso de masa y fuerza, otros serán simples términos funcionales a una teoría, tal como la noción de equilibrio en Piaget o, finalmente, algunos denotarán objetos reales, aunque no perceptibles, como lo son los átomos o los electrones. Pienso que es el segundo de los casos el que corresponde al concepto de valor, $y$ es lo que intento demostrar en este trabajo: al igual que número en las matemáticas, valor es un concepto teórico en la teoría de Marx, por lo tanto, imprescindible en ella, definible por teoría de conjuntos, pero no una sustancia como pensaba Marx.

Conocemos la respuesta de Russell: la correspondencia biunívoca entre dos conjuntos, permite decir que tienen igual número, $\mathrm{y}$ definir un número como el conjunto de todos los conjuntos que tienen ese mismo número.

En nuestro caso, teniendo definidos los conjuntos de chaquetas y varas de tela, lo que ponemos en correspondencia biunívoca no son la chaqueta y la tela, como no poníamos en correspondencia al Mariscal Ney con San Pedro, sino las horas de trabajo invertidas en producir las chaquetas y la tela. Los mariscales y los apóstoles son elementos del conjunto. Las horas son los elementos de los conjuntos marxis tas. Estos son los que pongo en correspondencia: los elementos. Una vez que pude ponerlos en correspondencia, puedo decir, en el primer caso, que tienen igual número; en el segundo, que tienen igual valor. ¿Qué será entonces valor? Contestaremos ahora como lo hizo Russell: es aquello que tienen los conjuntos que tienen igual valor. Una conceptuación que culmina cuatro etapas teóricas: primero, el trabajo indiferenciado del que nos habla Marx; luego, su cuantificación en horas de trabajo, para pasar de allí a definir igual valor, y finalmente, sentar la noción de valor. Existe aquí una maniobra extra que no figura en la definición de número, y es la función que conceta el conjunto temporal con el conjunto mercancía. 
Expresado en forma tosca, Marx dice lo siguiente:

$t$ horas de trabajo $\left\{\begin{array}{ll}\mathrm{y} \text { mercancías }=\mathrm{f} & (\mathrm{t}) \\ \mathrm{x} \text { mercancías }=\mathrm{f}_{1} & (\mathrm{t})\end{array}\right\}$ igual valor

Se trata de una función en los números reales, que va del dominio temporal al codominio de las mercancías, una función distinta para cada tipo de mercancía, $f$ para $y, f_{1}$ para $z$, y que determina la cantidad de mercancía específica que corresponde a $t$ horas de trabajo; esta función varía con el desarrollo de las fuerzas productivas, de forma tal que a mayor desarrollo de la fuerza productiva, la magnitud asignada en el conjunto temporal, y expresada en horas de trabajo, disminuye para la misma cantidad de mercancías. Si el número de horas trabajadas para producir $x$ y $y$ mercancías es igual, éstas tienen igual valor.

Más formalmente:

$$
\begin{aligned}
& \{\mathrm{M}\}=\mathrm{f}_{\mathrm{M}}\left(\mathrm{t}_{\mathrm{M}}\right) \\
& \{\mathrm{N}\}=\mathrm{f}_{\mathrm{N}}\left(\mathrm{t}_{\mathrm{N}}\right)
\end{aligned}
$$

$\star M$ tiene el mismo valor que $N$ sii $t_{M}=t_{N}$

Según Marx, y lo hemos seguido escrupulosamente, el tiempo socialmente necesario para la producción de un objeto, una mercancía, es la medida de su valor.

El valor es, entonces, una función de mercancías en tiempos.

Sea $m$ la clase de todas las mercancías.

$$
\mathrm{V}: \mathrm{P}(\mathrm{m}) \rightarrow \mathrm{T}
$$

La definición de valor se puede hacer, entonees, por medios conjuntísticos, en vez de hacerlo con una ontología sustancialista à la Aristóteles. El valor es un conjunto.

¿Truco de prestidigitación? ¿Respuesta insatisfactoria? Depende de los distintos puntos de vista. Con la constatación empírica del intercambio, Marx construye su ecuación, que 
ahora fundamento por teoría de conjuntos, sin recurrir a ninguna "metafísica oscura", limpiamente, legítimamente. Resulta más elegante y satisfactorio aceptar y trabajar teoría de conjuntos que objetividades fantasmales, sustancias gelatinosas.

Paso a contestar una objeción previsible: Mach define igual masa refiriéndose exclusivamente a conceptos cinemáticos, que son observacionales. ¿No podría hacerse algo semejante con valor? Opino que no; es discutible incluso si Mach hizo lo que pensaba que hacía, definir con términos cinemáticos puros a masa; pareciera que no es posible, al menos adecuadamente. Los intentos de definir por términos observacionales los términos teóricos han fracasado. Mi propia empresa epistemológica, en coincidencia con la filosofía de la ciencia de los últimos 30 años, no consiste en eliminarlos, tal como lo hacía Mach, aunque sin mencionarlo expresamente, sino en mantenerlos como tales en el seno de las teorías en las que son funcionales.

Una definición por teoría de conjuntos me permite mantener el status teórico del concepto valor.

En este contexto de fundamentación de la teoría del valor, ¿cómo podemos entender la cuantificación del valor, el último paso a elaborar con respecto a esta noción teórica?

Escribiré una ecuación de igualdad múltiple que espero arroje claridad en la cuestión:

20 varas de tela $=1$ chaqueta $=1 \mathrm{~kg}$ de hierro $=\mathrm{x}$ cantidad de trabajo

En la ecuación, ha sido puesto un signo igual entre chaquetas, tela, hierro, que son con las que Marx ilustraba su punto de vista, $y$ además cantidad de trabajo. Fste último agregado permite observar el hecho de que para Marx, el trabajo humano, bajo el régimen de producción de mercancías, es una mercancía más; como también entender que es uno más de los conjuntos que tienen el mismo valor. Tela, chaqueta, hierro y trabajo, en la ecuación propuesta, tienen el mismo valor. La maniobra, a continuación, es similar a la de Mach con respecto 
a masa: asignar un valor numérico a una masa para poder establecerla en los demás. En las mercancias-objetos, el valor permanece velado. En la mercancía-trabajo, el valor puede cuantificarse: la cantidad de trabajo puede medirse por el tiempo de trabajo socialmente necesario invertido. $Y$ conste que estamos ante una nueva entidad teórica: Marx no habla del tiempo real invertido en una mercancía necesaria, sino del tiempo necesario socialmente para producir las mercancías que tienen igual valor. La cuantificación del valor, entonces, depende de medir el tiempo de trabajo del que se pueda predicar que tiene el mismo valor que las mercancías del mismo valor.

No es mi intención formalizar toda la teoría del valor. Sé que la teoría de Marx tiene una lógica que lleva a considerar los demás factores que analiza más adelante.

Como buena teoría, sus afirmaciones forman un todo, del que sólo a los fines del análisis es posible desgajar un sector.

Quisiera sin embargo, poner dos últimos conjuntos en la larga, infinita, secuencia de igualdades entre mercancías:

$x$ mercancía $C=y$ horas de trabajo $=g$ mercancías necesarias para reponerlo y reproducirlo $=\mathrm{z}$ precio. ${ }^{1}$

Estas nuevas igualdades no son gratuitas, figuran en la obra de Marx tal como aquí las escribo. Con respecto al dinero, la igualdad puesta por Marx es: 20 varas de lienzo $=2$ onzas de oro (p. 86); para encontrar el conjunto tercero es necesario llegar al capítulo $\mathrm{V}$, en el que la forma es una explicitación

1 Me refiero a cada miembro de la igualdad como conjunto, ya que entiendo que cada uno de ellos es representativo de un conjunto integrado por todos los miembros que poscen las mismas características. Así, cuando Marx escribe 20 varas de tela $=1$ chaqueta, no se está en rigor refiriendo a estas 20 varas y a esta chaqueta en particular, sino a todos los conjuntos, cuyos miembros scan una chaqueta $y$, similarmente, a todos los conjuntos, cuyos miembros sean 20 varas de tela. De esta manera es factible la correspondencia biunívoca entre cada uno de estos miembros, una chaqueta y 20 varas. Lo mismo es válido para el resto de los miembros de la igualdad. No se trata de 9 horas de trabajo, sino de los conjuntos de 9 horas de trabajo. 
del tiempo de trabajo necesario para producir y reproducir la fuerza de trabajo.

Vemos ahora con nitidez, que también tienen el mismo valor que las mercancías consideradas, el precio que se paga por ellas y las mercancías con las cuales se va a reponer y reproducir la fuerza de trabajo socialmente consumida.

La ecuación marxista hace resaltar el error de la ley del valor de Diederich y Fulda: no se trata de una función que lleva de las horas de trabajo al valor, y de allí a una nueva función sobre los números reales, al precio. Es una única función de correspondencia biunívoca entre todos los conjuntos, de los que horas de trabajo y precio son apenas dos, que nos va a servir para definir, junto con Marx, la noción de igual valor.

Dos errores adicionales de la formulación de Diederich y Fulda: si bien tratan al valor como el resultante de una función de horas de trabajo sobre el valor, no especifican ninguna magnitud de valor; las únicas magnitudes en su ley son las horas de trabajo y el precio; el valor no posee magnitud, lo cual es bastante sorprendente si se lo define por una función sobre los números reales. La segunda objeción, que resulta de la eliminabilidad de la noción de valor por una función que vaya desde las horas de trabajo al precio, no significa que estos autores no le concedan status de término teórico al valor, sino que lo tratan como un término teórico de bajo nivel, similar a Peso Específico, que puede ser reemplaza do sin pérdida por la relación Peso/Volumen, y no como el término teórico complejo que realmente es, próximo a masa o fuerza en niveles de complejidad, pues, como estos términos, no puede ser eliminado por ninguna función entre términos no teóricos.

La determinación de la magnitud de la función valor en la teoría de Marx, teniendo en cuenta la ecuación compleja de igualdad que establecí siguiendo el texto de El Capital, se realiza tomando como unidad de valor cualquiera de los términos que figuran en la igualdad, sean chaquetas, varas de tela, onzas de oro u horas de trabajo; en la terminología de Marx, cualquiera de ellos puede ser equivalente de los demás, 
es decir, medida de valor. El valor, por lo tanto, se puede medir en chaquetas, oro o tiempo de trabajo, es indiferente a la teoría cuál medida se use; lo único que se le exige, como condición de restricción similar a la que rige a las medidas de términos teóricos de la física, es que sea de igual magnitud en cualquier aplicación de la teoría en la que vuelva a aparecer igual cantidad de mercancía. Aunque esto sea así desde el punto de vista teórico, pragmáticamente resulta más cómodo cuantificarlo con dinero o con tiempo de trabajo.

Formalmerte podemos asignar valor a una mercancía en dinero de la si ?uiente manera:

Sea $p \in m$ la mercancía que consiste en una moneda de 1 peso.

Entonces, convenimos en asignar

$$
V(\{p\})=1 .
$$

Fs decir, por definición, el valor de una moneda de un peso será igual a la unidad de tiempo socialmente necesario.

Para todos los conjuntos de mercancías $M \subset m$ tales que $M$ tiene el mismo valor que $\{\mathrm{p}\}$ (en el sentido de $\star$, p. 39, que decía: $M$ tiene el mismo valor que $N$ sii $t_{M}=t_{N}$ ), se sigue que

$$
\mathrm{V}(\mathrm{M})=\mathrm{l} .
$$

O sea:

$$
\{M\}=f_{M}(1) .
$$

Para aquellos conjuntos de mercancías $\mathrm{N}$ para los que necesitamos el doble de tiempo que para producir un peso, se sigue que

$$
V(N)=2,
$$

y así sucesivamente. 
De esta forma se ha metrizado el valor con dinero, que es así una manera privilegiada, sólo por razones de facilidad de manejo práctico y contable, de fijar la escala que mide el valor.

Incidentalmente, estas apreciaciones sobre la metrización del valor borran una vieja objeción metodológica contra la teoría marxista, que consiste en afirmar que no es posible distinguir entre el precio y el valor, o lo que es lo mismo, que en todos los lugares en que la teoría pone valor, en su uso se lee precio, lo que tornaría superfluo aquel concepto. En efecto, no pueden distinguirse, pues el precio expresa en su unidad el tener el mismo valor que una chaqueta o 20 varas de tela; pero esto no elimina la noción de valor, que sigue apareciendo en el resto de los conjuntos de la igualdad que se establece entre todas las mercancías que tienen el mismo valor, y en las sucesivas aplicaciones y expansiones de la teoría del valor que nos propone Marx en El Capital (plusvalía, cuota de ganancia, etcétera): el precio es sólo una medida cómoda que no sustituye ningún concepto teórico de El Capital.

Leer puntualmente a un autor, aceptar que las palabras juegan un rol funcional en el escrito, es esencial en los análisis de la filosofía e historia de la ciencia. Hacerlo nos permitió ubicar un nudo problemático en la fundamentación expuesta por Marx. El propio tex to nos sugirió las vías metodológicas de resolución. La reconstrucción parcial propuesta arroja luz sobre el pensamiento íntimo de Marx, y las enormes dificultades teóricas y de fundamentación que afrontó. Las herramientas forjadas a más de un siglo de su obra, permitieron cortar este nudo problemático.

\section{BIBLIOGRAFÍA}

Althusser, Louis, La revolución teórica de Marx, Siglo XXI, México, i978. Para leer El Capital, Siglo XXI, México, 1978.

Diederich, Werner, Hans Fulda, Estructuras sneedianas en El Capital de Marx, IIF, UNAM, México, 1981.

Newton, Isaac, Principia, University of California Press, EEUU, 1962.

Frege, Goltlob, Los fundamentos de la aritmética, IFF, UNAM, México, 1972. 
Marx, Karl, El Capital, Siglo XXI, México, 1979.

Moulines, Ulises, Exploraciones metacientlficas, Alianza, Madrid, 1982. "La génesis del positivismo en su contexto científico", Diánoia, México, UNAM-FCE,
1975 .

Russell, Bertrand, Obras completas, Ciencia y filosofía, Aguilar, España, 1973.

Sneed, Joseph, The Logical Structure of Mathemathical Physics, Reidel, Holland, 1979. 
SUMMARY

\section{MARX S MATERIALISM}

Marx has always been considered a pure materialist; there has never been any doubt about this point.

However, in the first chapter of Das Kapital, "work" is a strange substance, different from the material nature of things, hidden in goods, called by Marx "ghost matter, gelatinous matter, almost no matter at all". So, in goods there are, together, two kinds of substances: the matter of the objects, and the ghost matter, put there by the work that became itself a matter, and that remains even when you take off all the matter with which goods are made. The ontological monism of Marx -all things are matter-, stands postulating a very suspicious gelatinious matter.

Why does Marx behave in this way?

Mainly, to lay the foundations of the theoretical concept of "value", essential to his economic theory. He makes a substantialistic foundation of "value", thinking, with all the scientists of his time, that theoretical constructs have a substantial base. Even the image "gelatinous" is taken from physics: we might remember that the ether was equally gelatinous.

This problem is solved by Diederich and Fulda's formal reconstruction of value law, in which is no needed to say any thing about the kind of substance value is. Let us see how they do it:

$$
\text { (Value Law) } z \star v \star p
$$

$z$ is the time of work that is needed to make one product

$v$ is the value

$p$ is the price of that product

$\star$ is a function that determines the value once the time is known, and then determines the price.

The Value Law says that if one knows the time needed to make one product, there is a function that determines the value, and once that the value is determined, there is a function that determines the price. The substantial quality of value is eliminated.

But, in this reconstruction, value itself is eliminable because it is possible to put a composit function $\star$, and take off the formula the "value", in this way:

$$
\text { VL) } \mathrm{z} \star \star \mathrm{p}
$$

And this is possible because in Diederich and Fulda's reconstrution, even when "value" appears in the formula as a result of a function bet- 
ween "time" and "price", there is no numerical value of "value"; there is only scales of "time" and "price".

If it is so, Marx's theory is changed so deeply in this reconstruction, that Marx would not have accepted it. "Value" is a construct that can not be dismissed from the theory without eliminating the theory itself.

Let us see first how Marx states the problem. Surprisingly, he does not try to define "value", but "equal value", as it appears when two goods are exchanged in the market.

The problem he tries to explain, is how is it possible that:

1 quarter of wheat $=1$ quintal of iron.

Marx answers, following Aristotle, that they must have something in common: a common substance, a substance created by human work, the value.

Mach does the same when he defines first "equal mass" in his reconstruction of classic mechanics, and then defines "mass", showing that the way Marx goes is legitimate, and that in this direction no common substance is needed.

Some years later, Frege and Russell, trying to define "number", stated that sets have "equal number" when it is possible to put their elements in liunivocal correspondance. "Number" is tice set of all sets that have "equal number".

In the same way, the symbol $\Leftrightarrow$ (equal) that is put between two products when they are exchanged, needs no common substance. The only thing we need is to state a relationship of biunivocal correspondence between the elements of these two sets. From that point on, we can stand that there is a function in real numbers that goes from the set of time to the set of products, a different function for each kind of product, and that this function determines the quantity of a certain product that is related to thours of work; these functions change, as Marx said, with the development of the productive forces. The time socially necesary to produce an object is the measure of its value.

In a formal way:

$$
\begin{aligned}
& M=f_{M}\left(t_{M}\right) \\
& N=f_{N}\left(t_{N}\right)
\end{aligned}
$$

$M$ has the same value as $N$ if and only if $t_{M}=t_{N}$.

And the claim is that "value" is the set of all sets that have the same value, in a form that resembles the Frege-Russell solution to "number".

The set definition of "value" is possible, instead of defining it by means of an obscure metaphysical substance.

Marx's methodologic problem is solved. 